\section{Avaliação da situação do aleitamento materno em menores de um ano de idade no Município de São Paulo, Brasil, em 2008}

\author{
Infant breastfeeding prevalence in the city \\ of São Paulo, Brazil, 2008
}

\author{
Evaluación de la situación de la lactancia materna \\ en menores de un año de edad en el Municipio \\ de São Paulo, Brasil, en 2008
}

Lilian dos Santos Rodrigues Sadeck 1,2 Cléa Rodrigues Leone 1,2

\footnotetext{
1 Área Técnica da Saúde da Criança e Adolescente, Secretaria Municipal de Saúde de São Paulo, São Paulo, Brasil. 2 Faculdade de Medicina, Universidade de São Paulo, São Paulo, Brasil.

Correspondência L. S. R. Sadeck Área Técnica da Saúde da Criança e Adolescente, Secretaria Municipal de Saúde de São Paulo. Rua Dr. Augusto de Miranda 1092, apto. 83, São Paulo, SP 05026-001, Brasil.

liliansadeck@uol.com.br
}

\begin{abstract}
This cross-sectional epidemiological study based on AMAMUNIC aimed to evaluate infant breastfeeding (BF) prevalence and duration in São Paulo, Brazil. Sample size was based on a cluster calculation, selecting 35 primary care units and 35 infants ( $<1$ year of age) per unit. A total of 1,424 interviews with mothers were performed (56 items on feeding in the previous 24 hours) in August 2008, subsequently analyzed according to residential areas: Central West, East, North, Southeast, and South. Prevalence of exclusive breastfeeding (EBF) was 52\% up to three months and 39\% up to six months, as follows: $44 \%$ (Central West), 57\% (East), 62\% (North), 43\% (Southeast), and 48\% (South) up to three months and 36\% (Central West), 46\% (East), 39\% (North), 36\% (Southeast), and 33\% (South) up to six months. Prevalence of EBF according to infant age was $58 \%$ in the first month, $50 \%$ in the second and third, and $13 \%$ in the sixth. In conclusion, EBF prevalence up to six months is still heterogeneous in the city, suggesting the need for programs to encourage BF that are differentiated according to region.
\end{abstract}

Breast Feeding; Infant; Prevalence

\section{Resumo}

Estudo epidemiológico, transversal, baseado no Projeto AMAMUNIC. Foi desenvolvido com o objetivo de avaliar a situação de aleitamento materno (AM) - prevalência e duração - na cidade de São Paulo, Brasil. Realizou-se cálculo por conglomerados do número de entrevistas a serem realizadas. Sortearam-se 35 UBS e 35 crianças menores de um ano, por unidade. Foram realizadas 1.424 entrevistas (56 itens sobre alimentação nas últimas 24 horas) com os responsáveis de crianças menores de um ano, em agosto de 2008, as quais foram analisadas de acordo com as regiões de residência: centro-oeste, leste, norte, sudeste e sul. A prevalência de AM exclusivo (AME) foi $52 \%$ até três meses e $39 \%$ até seis meses, sendo 44\% (centro-oeste), 57\% (leste), 62\% (norte), $43 \%$ (sudeste) e 48\% (sul) até três e 36\% (centrooeste), 46\% (leste), 39\% (norte), 36\% (sudeste) e $33 \%$ (sul) até seis meses. A prevalência de AME de acordo com idade foi de $58 \%$ no primeiro mês, $50 \%$ no segundo e no terceiro e 13\% no sexto mês. Concluiu-se que a prevalência de AME até seis meses ainda é heterogênea na cidade, sugerindo a necessidade de programas de incentivo ao AM diferenciados entre as regiões.

Aleitamento Materno; Lactente; Prevalência 


\section{Introdução}

As vantagens da amamentação têm sido amplamente documentadas na literatura. Considera-se que o aleitamento materno (AM) associa perfeitamente três elementos fundamentais - alimentação, saúde e cuidados, constituindo-se na melhor alternativa para a nutrição das crianças após o nascimento. O conhecimento sobre AM é necessário para definição de políticas públicas de proteção, promoção e apoio a essa prática.

Nos Estados Unidos 1, em 2005, as taxas de $\mathrm{AM}$ até seis meses eram de $45 \%$ e, até 12 meses, $25 \%$. Foram, então, propostos objetivos para o Healthy People 2010, visando a aumentar as taxas de AM para $50 \%$ aos seis meses e $25 \%$ até 12 meses.

Em nosso país, a primeira estimativa nacional, baseada em metodologia científica mais criteriosa sobre a frequência do aleitamento materno exclusivo (AME), foi a da Pesquisa Nacional sobre Mortalidade Infantil e Planejamento Familiar realizada em 1986 (PNMIPF/1986) 2. Nessa investigação, o AME era de 3,6\% entre as crianças brasileiras com zero a quatro meses.

Em 1999, o Ministério da Saúde 3, em inquérito populacional, verificou heterogeneidade entre as capitais e regiões do país. A prevalência de AME foi de 35,6\% em menores de quatro meses, enquanto no Município de São Paulo foi de 24,9\%.

O objetivo deste estudo é verificar a prevalência de AME e aleitamento materno predominante (AMP) em crianças menores de três e de seis meses e AM entre as de nove e 12 meses, no Município de São Paulo e suas regiões, comparando os resultados com os da pesquisa de 1999.

\section{Metodologia e casuística}

Estudo epidemiológico, transversal, baseado no Projeto AMAMUNIC do Instituto de Saúde de São Paulo 4,5,6. O cálculo do número de questionários a serem aplicados foi o de amostra por conglomerados. Os planos amostrais foram elaborados com base no número de postos de vacinação na cidade; a estimativa do número de crianças menores de um ano que seriam vacinadas em cada posto foi feita com base nas planilhas de campanhas de vacinação de 2007. Na primeira fase, realizou-se o sorteio das unidades básicas de saúde (UBS), com probabilidade proporcional ao tamanho dos conglomerados; no segundo estágio, sortearam-se as crianças em cada posto, de forma sistemática. A amostra desenvolvida foi considerada equiprobabilística ou autoponderada. Foram sorteadas 35 UBS e 35 crianças menores de um ano para as entrevistas em cada unidade, incluindo os postos volantes. Estimouse um total de 1.225 entrevistas. Para a coleta de dados, foram treinados 57 supervisores e 357 entrevistadores.

O questionário aplicado a pais/responsáveis legais de crianças menores de um ano de idade, na segunda fase da Campanha Nacional de Vacinação contra Poliomielite em agosto de 2008, continha 56 questões sobre o consumo de leite materno, outros tipos de leite, água, sucos ou chás e outros alimentos nas últimas 24 horas; sobre a amamentação, quando iniciada e frequência, além de perguntas sobre local e condições de nascimento. Questionou-se sobre uso de chupeta e presença da mãe em casa, por licença do trabalho ou não, condições socioeconômicas e escolaridade materna ${ }^{7}$.

Após digitação dos dados, com o apoio da equipe de informática do Instituto de Comunicação e Informação Científica e Tecnológica em Saúde, Fundação Oswaldo Cruz (ICICT/Fiocruz), foi realizada a análise relativa ao município e região de residência da criança: centro-oeste, leste, norte, sudeste e sul. A avaliação da prevalência do AM seguiu as recomendações da Organização Mundial da Saúde (OMS) 7:

- AME: oferta de leite materno sem quaisquer outros líquidos ou alimentos, exceto medicamentos;

- AMP: oferta de leite materno e apenas água ou chá;

- AM: oferta de leite materno e qualquer outro líquido ou alimento.

Calculou-se a prevalência de AME, AMP até três e até seis meses e de AM entre as crianças de nove a 12 meses no município e regiões de residência da criança. Compararam-se os resultados aos da I Pesquisa Nacional sobre Aleitamento Materno em 19993.

Os acompanhantes deram seu consentimento verbal para a aplicação do questionário. A pesquisa foi aprovada pelo Comitê de Ética e Pesquisa da Secretaria Municipal de Saúde de São Paulo (protocolo 001/08).

\section{Resultados}

Foram realizadas 1.557 entrevistas $(127 \%$ das 1.225 propostas), sendo excluídas $133(8,6 \%)$ por recusa em participar; foram digitadas 1.424 , mas analisadas 1.398 , por haver dados incompletos ou serem de não residentes no município. A distribuição nas regiões foi de $19 \%$ na centro-oeste, $21 \%$ na leste, $15 \%$ na nordeste, $21 \%$ na sudeste e $24 \%$ na sul.

A prevalência de AM aos seis meses, considerando-se as crianças em AME e AMP, foi de 54\%, 
sendo de $51 \%$ na região centro-oeste, $63 \%$ na leste, $49 \%$ na norte, $48 \%$ na sudeste e $50 \%$ na sul.

A prevalência de AME foi de $52 \%$ até três meses e $39 \%$ até seis meses de idade (Figura 1). O AMP foi de $17 \%$ até três meses e $15 \%$ até seis meses, variando de $13 \%$ a $19 \%$ até três meses e $10 \%$ a $17 \%$ até seis meses.

A prevalência de AME de acordo com a idade encontra-se na Figura 2. Observa-se que a maior queda da prevalência ocorreu entre o quinto e o sexto meses de idade, com $123 \%$ de redução relativa.

A prevalência de AME até quatro meses de idade foi de $49,8 \%$, apresentando um aumento relativo de $100 \%$ em relação à encontrada na pesquisa de 1999, que foi de 24,9\%. Das 316 crianças com idade entre nove meses e um ano, 48,8\% ainda mantinham o AM, enquanto, na pesquisa de 1999, a prevalência foi de 32,9\%.

\section{Discussão}

O AM promove uma oferta adequada e essencial de nutrientes para o crescimento e desenvolvimento das crianças, além de proteger contra infecções e outras doenças, aumentando as chan- ces de sobrevida. A OMS, baseada em evidências científicas, recomenda o AME até seis meses e sua continuidade, com suplementação de outros alimentos, até os dois anos de idade ?.

Cada vez mais, o aleitamento materno tem sido aceito e praticado pelas mães vinculadas à rotina dos serviços de saúde que prestam assistência à criança. $\mathrm{O}$ aumento progressivo das taxas de aleitamento tem sido relacionado à implantação e implementação de políticas públicas direcionadas para o incentivo ao aleitamento materno 8,9,10,11.

A necessidade de se avaliar periodicamente a situação do AM em cada local estimulou o desenvolvimento de instrumentos com metodologia adequada e de fácil aplicabilidade para levantamentos epidemiológicos. O Projeto AMAMUNIC, desenvolvido pelo Instituto de Saúde de São Paulo, é um instrumento comprovado para esses levantamentos.

Este estudo avaliou a prevalência de AM até seis meses e entre nove e 12 meses, encontrando valores acima da meta estabelecida pela Health Global 2010, de 50\% e 25\%, respectivamente. Também se verificou um aumento da prevalência de AME em relação à pesquisa anterior. No entanto, em menores de seis meses de idade a

Figura 1

Prevalência de aleitamento materno exclusivo (AME) até três meses e até seis meses de idade, de acordo com a região de residência no Município de São Paulo, Brasil.

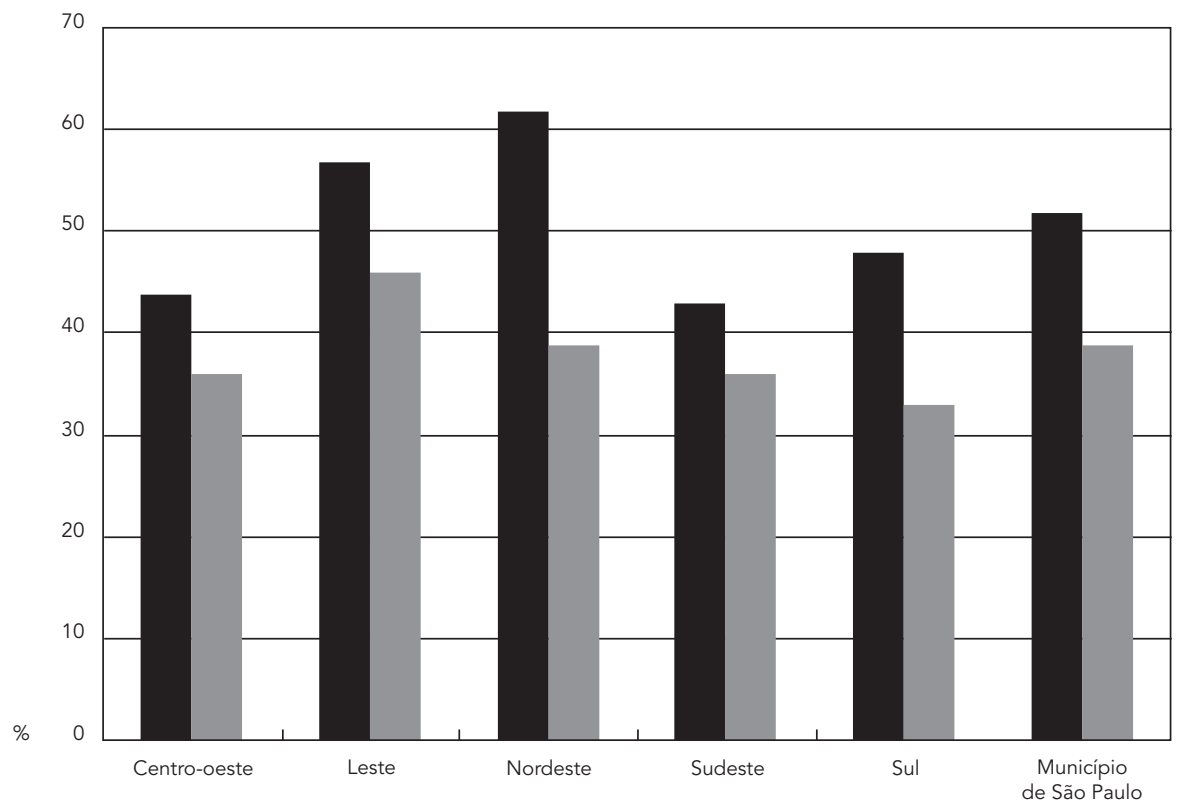


Prevalência de aleitamento materno exclusivo (AME) de acordo com a idade até o sexto mês de vida, no Município de São Paulo, Brasil.

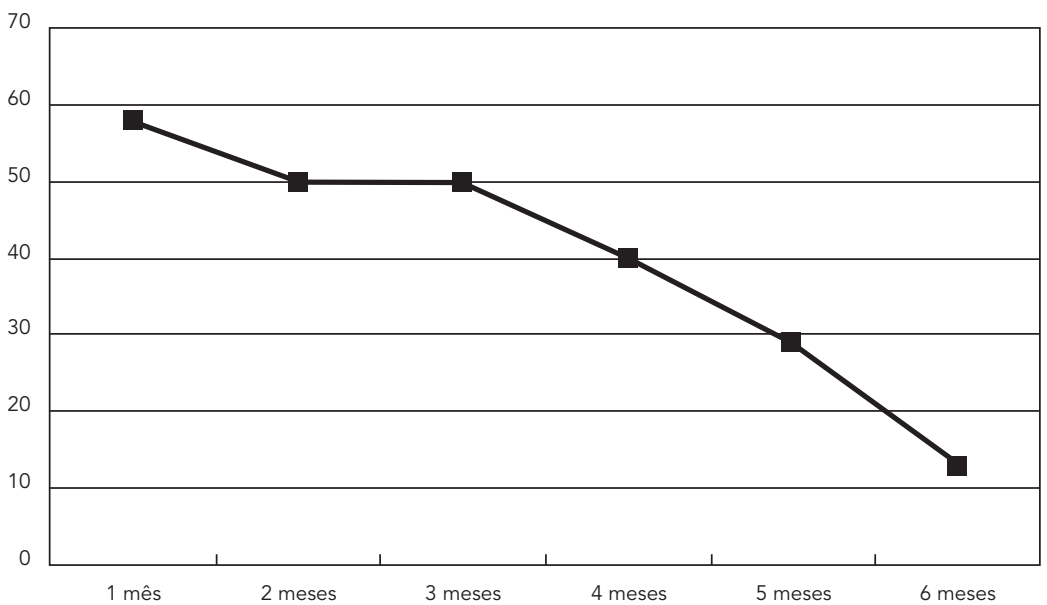

situação ainda é inadequada, sendo considerada ruim pelos critérios da OMS.

A monitorização da evolução da prevalência de AME nos primeiros seis meses de vida mostra que, até o primeiro trimestre, esta permanece razoavelmente estável, porém, após esse período, ocorre uma redução progressiva. Essa evolução pode estar relacionada às condições de vida dessas famílias, cujas mães, muito frequentemente, trabalham fora e têm necessidade de retornar a suas atividades por razões econômicas e também para a manutenção de seus postos de trabalho 12 .

Embora o presente estudo sinalize crescimento, ao longo do tempo, da prevalência de AME e AMP até os seis meses de idade, a literatura aponta uma grande variabilidade das taxas de AME aos seis meses, desde $62 \% 7$ até $13,3 \% 13$. O mesmo foi verificado na cidade em estudo, onde esses indicadores oscilaram entre $33 \%$ e $46 \%$ e de
$48 \%$ até $63 \%$, respectivamente, de acordo com local de residência.

Considerando-se a heterogeneidade do $\mathrm{Mu}$ nicípio de São Paulo, a variação da prevalência de AM entre as cinco regiões estudadas era esperada. A pesquisa identificou as regiões com menores taxas de AME e AM, indicando os locais onde deveriam ser desenvolvidos programas diferenciados de incentivo ao AM. De forma inversa, nas regiões em que as taxas de AM foram maiores, o conhecimento dos fatores responsáveis pelo sucesso dos programas pode se constituir numa ferramenta útil para que outras regiões possam obter melhores resultados.

A realização periódica de pesquisas sobre práticas alimentares em crianças menores de um ano de idade é um instrumento indispensável de avaliação de resultados de programas desenvolvidos, bem como de novas intervenções, mais adequadas a cada local. 


\section{Resumen}

Estudio epidemiológico, transversal, basado en el AMAMUNIC. Fue desarrollado con el objetivo de evaluar la situación de lactancia materna (AM) - prevalencia y duración- en la ciudad de São Paulo, Brasil. Se realizó un cálculo por conglomerados del número de entrevistas que se debían realizar. Se sortearon 35 UBS y se adscribieron 35 niños menores de un año por unidad. Fueron realizadas 1.424 entrevistas (56 items sobre alimentación en las últimas 24 horas) con los responsables de los niños menores de un año, en agosto de 2008, las cuales fueron analizadas de acuerdo con las regiones de residencia: centro-oeste, este, norte, sudeste y sur. La prevalencia de AM exclusivo (AME) fue de un 52\% hasta tres meses y un 39\% hasta seis meses, siendo hasta tres meses 44\% (centro-oeste), 57\% (este), $62 \%$ (norte), 43\% (sudeste) y $48 \%$ (sur), y un 36\% (centro-oeste), $46 \%$ (este), 39\% (norte), 36\% (sudeste) y 33\% (sur) hasta los seis meses. La prevalencia de AME de acuerdo con la edad fue de un 58\% en el primer mes, $50 \%$ en el segundo y en el tercero y un $13 \%$ en el sexto mes. Se concluyó que la prevalencia de AME hasta seis meses todavía es heterogénea en la ciudad, sugiriendo la necesidad de programas de incentivo al AM, diferenciados entre las regiones.

Lactancia Materna; Lactante; Prevalencia

\section{Colaboradores}

L. S. R. Sadeck e C. R. Leone contribuíram substancialmente na concepção, planejamento, análise e interpretação dos dados; na elaboração do rascunho e na revisão crítica do conteúdo. Participaram, ainda, da aprovação da versão final do manuscrito.

\section{Agradecimentos}

Programa Rede de Proteção à Mãe Paulistana, da Secretaria Municipal de Saúde de São Paulo (Coordenador Técnico: Celso de Moraes Terra; Grupo de Trabalho de Humanização da Assistência à Criança: Agenor de Freitas L. Junior, Bertille Ferreira, Camila Correa, Hitomi Hayashida, Lélia Cardamone Gomes, Lucilene Terrengui, Maria José G. Mattar, Olga Aparecida F. Caron, Regina Yoshihara, Sonia I. Venâncio, Zuleika Uehara; Coordenadores Regionais: Ana Lucia G. Demarchi, Ana Lucia P. Batista, Ana Maria S. Graseffe, Aparecida J. C. Gasques, Marília A. de Oliveira, Sonia Regina L. A. Prado).

\section{Referências}

1. Grummer-Strawn LM, Shealy KR. Progress in protecting, promoting and supporting breastfeeding: 1984-2009. Breastfeed Med 2009; 4:S31-9.

2. Leão MM, Coutinho DC, Recine E, Costa LAL, Lacerda AJ. O perfil do aleitamento materno no Brasil. In: Instituto Brasileiro de Geografia e Estatística; Fundo das Nações Unidas para a Infância, organizadores. Perfil estatístico de crianças e mães no Brasil. Rio de Janeiro: Instituto Brasileiro de Geografia e Estatística; 1989. p. 97-110.

3. Ministério da Saúde. Prevalência de aleitamento materno nas capitais brasileiras e no distrito federal. Brasília: Ministério da Saúde; 2001.

4. Silva NN. Amostragem probabilística: um curso introdutório. São Paulo: Edusp; 1998
5. Venâncio SI, Escuder MML, Kitoko P, Rea MF, Monteiro CA. Frequência e determinantes do aleitamento materno em municípios do Estado de São Paulo, 1998. Rev Saúde Pública 2002: 36:306-13.

6. Venancio SI, Monteiro CA. Individual and contextual determinants of exclusive breastfeeding in São Paulo, Brazil: a multilevel analysis. Public Health Nutr 2006; 9:40-6.

7. Division of Child Health and Development, World Health Organization. Indicators for assessing and young child practices: conclusions of consensus meeting held 6-8 November 2007. Washington DC: World Health Organization; 2008. 
8. Chudasama RK, Amin CD, Parikh YN. Prevalence of exclusive breastfeeding and its determinants in first 6 months of life: a prospective study. Online J Health Allied Sci 2009; 8(1). http://www.ojhas.org/ issue29/2009-1-3.htm.

9. Li R, Zhao Z, Mokdad A, Barker L, Grummer-Strawn L. Prevalence of breastfeeding in the United States: the 2001 National Immunization Survey. Pediatrics 2003; 111(5 Part 2):1198-201.

10. Venancio SI, Monteiro CA. A evolução da prática da amamentação nas décadas de 70 e 80. Rev Bras Epidemiol 1998; 1:40-9.

11. Yngve A, Kylberg E, Sjostrom M. Breast-feeding in Europe: rationale and prevalence, challenges and possibilities for promotion. Public Health Nutr 2001; 4:1353-5.
12. Leone CR, Sadeck LSR. Programa de Proteção à Mãe Paulistana. Fatores de risco associados ao desmame em crianças até 6 meses de vida no município de São Paulo. Rev Paul Pediatr 2012; 30:21-6.

13. Li R, Darling N, Maurice E, Barker L, GrummerStrawn LM. Breastfeeding rates in the United States by characteristics of the child, mother, or family: the 2002 National Immunization. Pediatrics 2005; 115:e31-7.

Recebido em 24/Jan/2012

Versão final reapresentada em 13/Set/2012

Aprovado em 03/Out/2012 